\title{
ACCURACY ANALYSIS OF THE FAST MULTIPOLE METHOD FOR THREE-DIMENSIONAL BOUNDARY VALUE PROBLEMS BASED ON LAPLACE'S EQUATION
}

\author{
ANDRÉ BUCHAU \\ Institute of Smart Sensors, University of Stuttgart, Germany
}

\begin{abstract}
The fast multipole method (FMM) is an established matrix compression technique for a fast and efficient solution of complex three-dimensional boundary value problems based on Laplace's equation using the boundary element method (BEM). Furthermore, the FMM significantly accelerates the postprocessing of the solved BEM problem. The foundation of the FMM is a series expansion of Green's function into spherical harmonics. Both the accuracy and computational costs are controlled by the order of this truncated series expansion. The FMM has been successfully applied to a plethora of engineering problems from multiple disciplines and its overall accuracy has been impressively proved. The basic numerical properties of the truncated series expansions of the FMM have been already investigated in detail but for point sources only. Here, I revisit the fundamentals of the FMM and analyze the relevant properties of spherical harmonics, the error of the truncated series expansions of the FMM, the influence of coordinate transformations of these series expansions and potential discontinuities of computed field values at the interfaces between the cubes of the hierarchical octree scheme of the FMM. In contrast to earlier publications, I analyze and visualize the field values of distributed single-layer sources instead of point sources inside the total computational domain of an octree cube with the help of three-dimensional plots of their field values and their related approximation errors. For these studies, I put a focus on the accuracy of the reversed FMM algorithm, which is very well suited for meshfree post-processing and which is introduced here in detail for the first time.
\end{abstract}

Keywords: Laplace's equation, boundary element method, fast multipole method, spherical harmonics, post-processing.

\section{INTRODUCTION}

The fast multipole method (FMM) has been originally developed by Greengard and Rokhlin [1] to compute particle interactions efficiently. They have shown impressively that the computational costs for a system of $N$ regularly distributed particles are reduced from $O\left(N^{2}\right)$ to $O(N \log N)$, if the well-known series expansion of $\frac{1}{4 \pi\left|\boldsymbol{r}-\boldsymbol{r}^{\prime}\right|}$ into spherical harmonics is applied in combination with a hierarchical octree scheme. They have applied a high order of the truncated series expansions to get accurate results due to the singular fields of the particles.

Nabors and White [2] have extended this approach to the solution of electrostatic field problems using an indirect formulation of the boundary element method (BEM). Later, an adaptive scheme for generalized BEM computations based on higher-order boundary elements and the Galerkin method has been introduced for electrostatic field problems [3]. In [4] it has been shown that the FMM competes very well with the adaptive cross approximation technique (ACA) and is furthermore applicable for a coupling of the BEM with the finite element method (FEM). In all these mentioned applications, the error caused by the FMM has been in the range of the total error of the resulting linear equation system and is barely visible in integral values like the capacitance coefficients of a multiconductor system [2]. 
Remarkably, despite the success of the FMM, a detailed analysis of the approximation error of the series expansions in the context of typical BEM integrals cannot be found in the literature. Hence, I present here a study of the approximation error of single-layer integrals as they occur in the context of indirect BEM formulations or as a part of the integrals of direct BEM formulations [5]. First, I revisit the properties of the used spherical harmonics and the multipole and local expansions of a single-layer source at a single boundary element. In contrast to particles, the scalar potential $u$ stays finite at the boundary element. My study includes evaluations of the potential or its gradient, which is the field strength, in the related octree cube. Particularly, I put a focus on the interfaces between two cubes to investigate potential discontinuities of normally continuous field values caused by the truncated FMM series expansions. This point is especially important for precise meshfree post-processing as it is required for instance for the design of magnets for nuclear magnetic resonance (NMR) applications [6]. The reversed FMM algorithm, which was firstly applied in [7], is the basis for the following error analysis of the FMM transformations. There, efficient transformation operators as presented in [8] are employed for accurate and fast transformations of the multipole and local coefficients. Finally, I draw my conclusions based on three-dimensional plots of the fields and their approximation errors to first show the numerical properties of the multipole and local expansions but second to support a reasonable use of the FMM in the context of the BEM or related applications.

\section{MULTIPOLE AND LOCAL EXPANSIONS}

The starting point of my study is Laplace's equation

$$
\Delta u=0,
$$

which is the fundamental partial differential equation of an electrostatic field problem based on the scalar electric potential $u$. The related Green's function for three-dimensional open space field problems is then

$$
G\left(\boldsymbol{r}, \boldsymbol{r}^{\prime}\right)=\frac{1}{4 \pi} \frac{1}{\left|\boldsymbol{r}-\boldsymbol{r}^{\prime}\right|}
$$

The point $\boldsymbol{r}^{\prime}$ describes a point on the boundary elements or the single-layer sources, respectively. The field values are computed at the evaluation point $\boldsymbol{r}$.

The basis of the FMM is the series expansion of eqn (2) into spherical harmonics $Y_{n}^{m}$ [9]

$$
\frac{1}{4 \pi} \frac{1}{\left|\boldsymbol{r}-\boldsymbol{r}^{\prime}\right|}=\left\{\begin{array}{ll}
\frac{1}{4 \pi} \sum_{n=0}^{\infty} \sum_{m=-n}^{n} \frac{r^{n}}{r^{\prime n+1}} Y_{n}^{m}(\theta, \phi) Y_{n}^{-m}\left(\theta^{\prime}, \phi^{\prime}\right) & r<r^{\prime} \\
\frac{1}{4 \pi} \sum_{n=0}^{\infty} \sum_{m=-n}^{n} \frac{r^{\prime}}{r^{n+1}} Y_{n}^{m}(\theta, \phi) Y_{n}^{-m}\left(\theta^{\prime}, \phi^{\prime}\right) & r^{\prime}<r
\end{array} .\right.
$$

$r, \theta$ and $\phi$ are the spherical coordinates of $\boldsymbol{r}$ and $r^{\prime}, \theta^{\prime}$ and $\phi^{\prime}$ are the spherical coordinates of $\boldsymbol{r}^{\prime}$, respectively. Note, that the imaginary part of the double sum in eqn (3) vanishes.

\subsection{Normalized spherical harmonics}

Depending on the intended application of spherical harmonics, different normalization approaches are used. Here, I use the normalization which has been introduced in [1], since it is very advantageous in the context of the FMM regarding computational costs 


$$
Y_{n}^{m}(\theta, \phi)=\sqrt{\frac{(n-|m|) !}{(n+|m|) !}} P_{n}^{|m|}(\cos \theta) \mathrm{e}^{\mathrm{j} m \phi} .
$$

$n$ is the degree of the spherical harmonics and $m$ is its order with $|m| \leq n . P_{n}^{m}(\cos \theta)$ are the associated Legendre functions and are computed using recurrence relations [9], [10].

The value of $Y_{0}^{0}(\theta, \phi)$ is constantly one. With increasing degree $n$, the number of lobes in a three-dimensional plot of $Y_{n}^{m}(\theta, \phi)$ increases, too. The direction of these lobes is adjusted by the order $m$. This is exemplarily depicted in Fig. 1 for the real part of $Y_{1}^{1}(\theta, \phi)$ and the imaginary part of $Y_{4}^{2}(\theta, \phi)$. The values of $Y_{n}^{m}(\theta, \phi)$ are additionally weighted with the factor $r^{n}$ in the series expansions in eqn (3). That means higher order spherical harmonics are especially relevant for large distances to the origin of the coordinate system (Fig. 2).
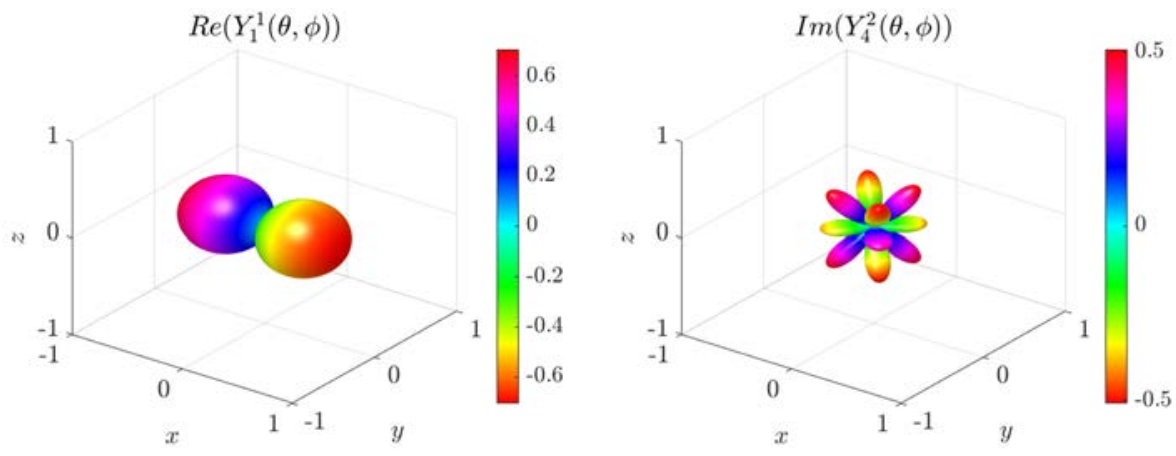

Figure 1: The real part of $Y_{1}^{1}(\theta, \phi)$ and the imaginary part of $Y_{4}^{2}(\theta, \phi)$.

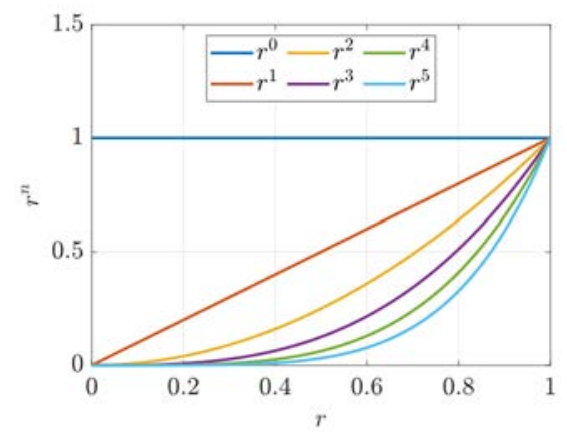

Figure 2: Values of the weighting factor $r^{n}$ within the normalized interval of $0 \leq r \leq 1$.

\subsection{Multipole expansion}

The truncated multipole expansion of order $L$ is obtained from eqn (3) for the case $r^{\prime}<r$

$$
u(r, \theta, \phi)=\frac{1}{4 \pi \epsilon_{0}} \sum_{n=0}^{L} \sum_{m=-n}^{n} \frac{Y_{n}^{m}(\theta, \phi)}{r^{n+1}} M_{n}^{m} .
$$

$\epsilon_{0}$ is the electric permittivity of vacuum and $u$ the potential of an electrostatic field problem. 
The single-layer charge density $\sigma$ on boundary elements inside a spherical domain around the origin of the coordinate system is summarized by the complex multipole coefficients

$$
M_{n}^{m}=\int_{A} \sigma(r, \theta, \phi) r^{\prime n} Y_{n}^{-m}\left(\theta^{\prime}, \phi^{\prime}\right) \mathrm{d} A^{\prime}
$$

The $M_{n}^{m}$ correspond to point sources of degree $n$ at the origin and are equivalent to a monopole, a dipole, a quadrupole and multipoles of higher degree [11]. A discussion of the accuracy of eqn (5) follows in Section 3 in the context of the reversed FMM algorithm.

\subsection{Local expansion}

The local expansion is the evaluation of eqn (3) inside a domain around the origin of a spherical coordinate system, which is for the case $r<r^{\prime}$

$$
u(r, \theta, \phi)=\frac{1}{4 \pi \epsilon_{0}} \sum_{n=0}^{L} \sum_{m=-n}^{n} r^{n} Y_{n}^{m}(\theta, \phi) L_{n}^{m}
$$

with the complex local coefficients

$$
L_{n}^{m}=\int_{A} \sigma(r, \theta, \phi) \frac{Y_{n}^{-m}\left(\theta^{\prime}, \phi^{\prime}\right)}{r^{\prime n+1}} \mathrm{~d} A^{\prime}
$$

The $L_{n}^{m}$ are not computed directly using eqn (8) but with transformations of the FMM algorithm indirectly with the help of the $M_{n}^{m}$.

Eqn (7) corresponds to a Taylor series expansion of the potential $u$ at the origin of the spherical coordinate system. The degree $n=0$ term represented by $L_{0}^{0}$ is equivalent to the potential at the origin and results in a constant potential inside the complete octree cube with its center at the origin of the spherical coordinate system. The three $n=1$ terms add linear terms to the function of $u$ in the considered domain. The value and sign of $L_{1}^{0}$ controls the linear dependency of $u$ in the $z$-direction, the real part of $L_{1}^{1}$ controls the linear dependency on the $x$-coordinate and the imaginary part of $L_{1}^{1}$ adds a linear term dependent on the $y$ coordinate (Fig. 3). With the help of the higher order terms $n \geq 2$, the function $u(x, y, z)$ inside the valid domain is further shaped and adapted to the original function of $u$. In Fig. 4 the results of the imaginary part of $L_{3}^{3}$ are shown. There, the potential $u$ in the FMM cube depends only on the $x$-and $y$-coordinates and is independent of the $z$-coordinate.
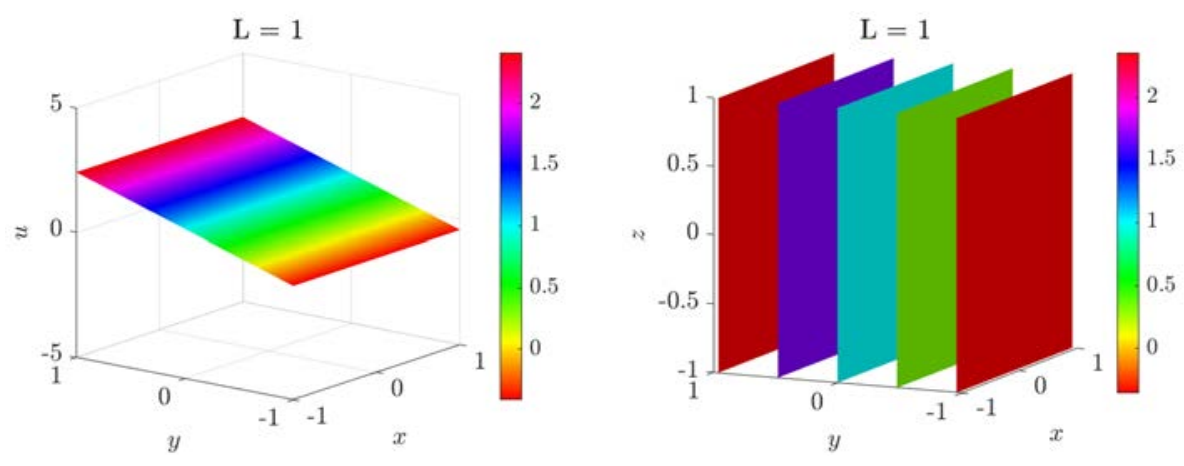

Figure 3: Potential $u$ in a horizontal slice and its isosurfaces controlled by $\operatorname{Im}\left(L_{1}^{1}\right)$. 

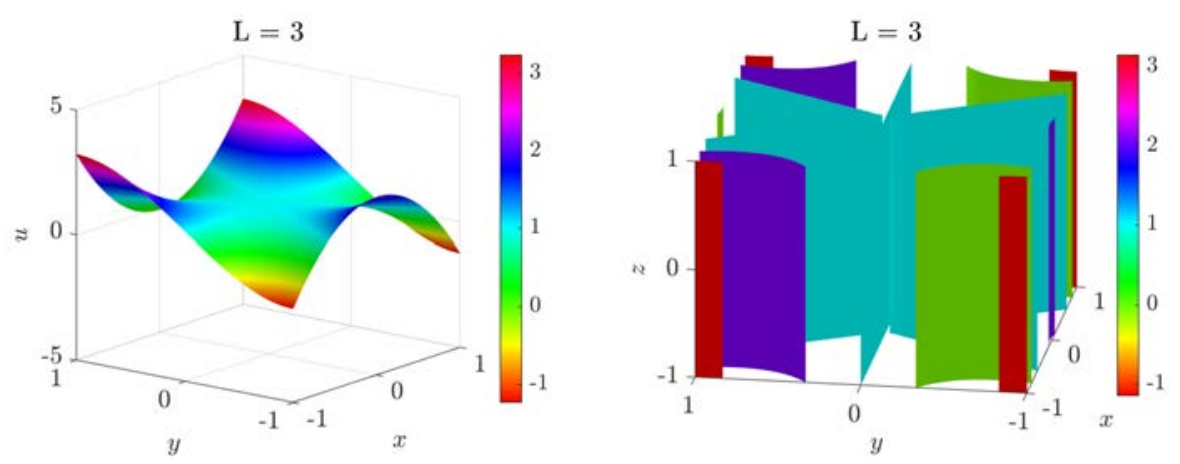

Figure 4: Potential $u$ in a horizontal slice and its isosurfaces controlled by $\operatorname{Im}\left(L_{3}^{3}\right)$.

It is worth mentioning that the product of $r^{n} Y_{n}^{m}(\theta, \phi)$ results in classical polynomial functions in Cartesian coordinates and is therefore well suited for an accurate approximation of scalar potentials $u(x, y, z)$, if there are no sources inside the considered evaluation domain.

\section{REVERSED FMM ALGORITHM}

The reversed FMM algorithm is perfect for post-processing and has been already successfully applied to the meshfree computation of field lines [7]. There, the idea that an FMM algorithm in reversed order is advantageous has been introduced in principle. Here, I present the reversed FMM algorithm in detail for the first time and analyze its numerical properties concerning the accuracy of its related series expansions and transformation operations.

\subsection{Post-processing based on the reversed FMM algorithm}

It is obvious to apply the FMM both to compress the matrix of the linear system of equations of the BEM and to improve its post-processing overall efficiency. However, there is a major difference between matrix compression and meshfree post-processing. In the first case, the position of all sources which are represented by the boundary elements is fixed and the field values are computed at fixed points, too [3]. Whereas an important property of meshfree postprocessing is that only the sources and their positions are known in advance and the evaluation points are determined during the post-processing [12]. That means the structure of the underlying octree scheme is changing during the post-processing and the focus moves there especially to an efficient evaluation of eqn (7) including a suitable computation of the $L_{n}^{m}$.

All steps of the reversed FMM algorithm are summarized schematically in Fig. 5. Each step has an equivalent step in the original FMM algorithm [1] but I reversed the order of the steps and added conditions for the execution of a step to accelerate meshfree post-processing.

The reversed FMM algorithm is initialized with an octree that includes all boundary elements as sources. Then, in step 1 the current evaluation point $\boldsymbol{r}_{e p}$, e.g. the next point of a field line, is added to the octree and its related cube $C_{e p}$ is created if necessary. $C_{e p}$ is identical to the cube in which the local expansion of step 2 must be evaluated. To compute field values based on eqn (7), the $L_{n}^{m}$ of $C_{e p}$ are needed. If the $L_{n}^{m}$ have been already computed within a previous step, e.g. for the preceding point of the field line, these $L_{n}^{m}$ are taken in step 3 and are evaluated in step 4 using eqn (7) or the derivative of eqn (7) with respect to the global Cartesian coordinates to determine flux densities, e.g. the electric field $\boldsymbol{E}=-\nabla u$. 


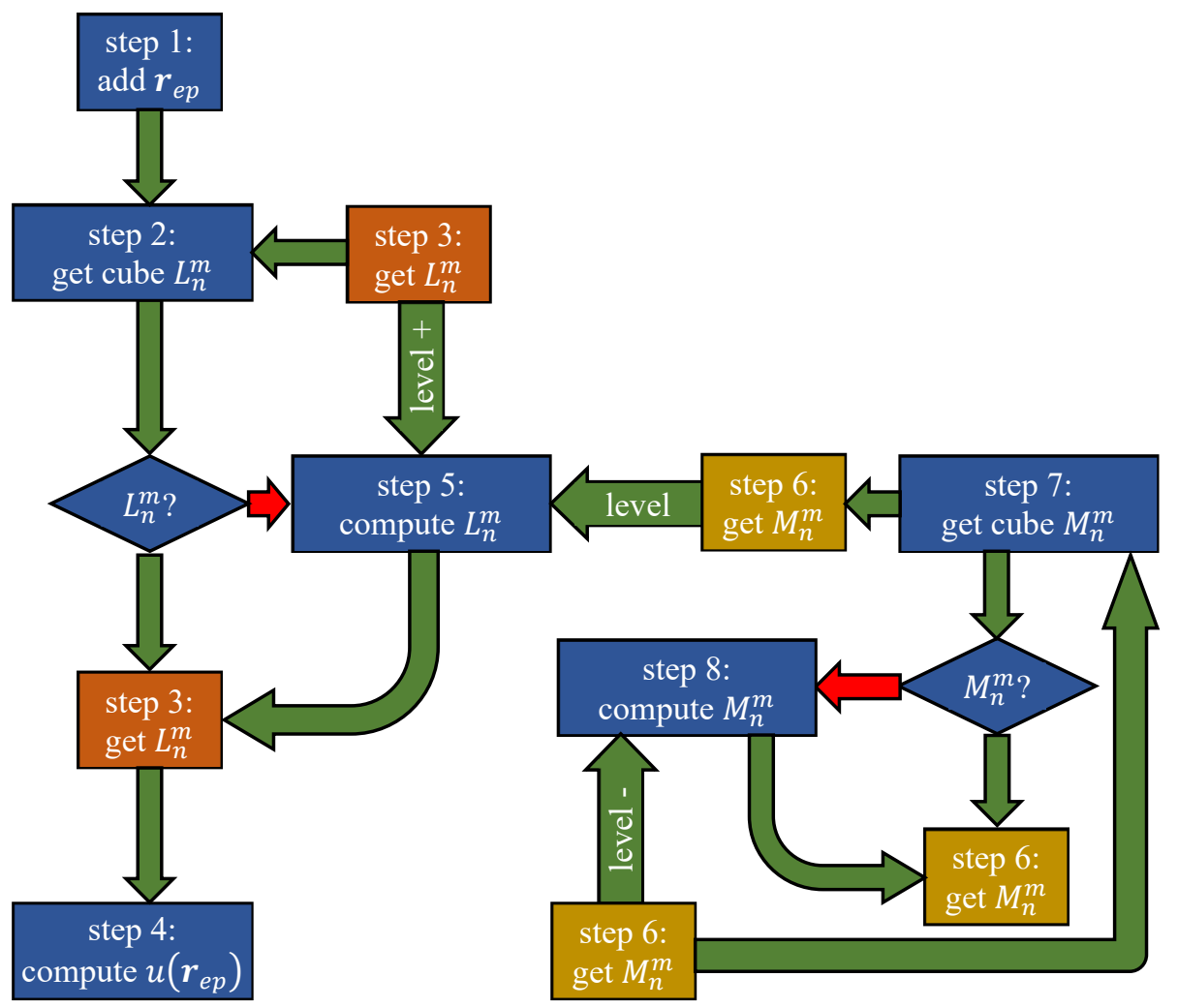

Figure 5: Computational steps of the reversed FMM algorithm.

Step 5 is only executed if the $L_{n}^{m}$ have not been computed within an earlier computation. The $L_{n}^{m}$ are computed from the related coefficients of the parent cube $C_{e p, p}$ of $C_{e p}$ at the next coarser level of the octree (level+). This is equivalent to step 3 which now redirects to step 2 but using $C_{e p, p}$ instead of $C_{e p}$. If this loop ends in step 5, the loop is repeated if necessary. Finally, step 3 is reached and the $\bar{L}_{n}^{m}$ of $C_{e p, p}$ are transformed into $L_{n}^{m}$ of $C_{e p}$

$$
L_{n}^{m}=\sum_{k=n}^{L} \bar{L}_{n}^{m} T_{l 2 l}(n, m, k)
$$

after a suitable rotation of the coordinate system to accelerate the computations [8]. The transformation coefficients $T_{l 2 l}(n, m, k)$ are computed once for each possible direction and modified for each octree level

$$
T_{l 2 l}(n, m, k)=T_{l 2 l}^{\text {direction }} T_{l 2 l}^{\text {root }} T_{l 2 l}^{\text {level }} .
$$

Additionally, the multipole coefficients $M_{n}^{m}$ of far-field cubes at the same level are determined (step 6) and added to the local coefficients of $C_{e p}$

$$
L_{n}^{m}=\sum_{k=m}^{L} M_{k}^{m} T_{m 2 l}(n, m, k) .
$$


Here, a rotation of the coordinate system is done first, too [8].

In step 7, cubes at the same octree level as $C_{e p}$ must be found which are far away from $C_{e p}$ to ensure the validity of the series expansions and which are not in a region that has been already considered in the far-field at a coarser octree level. For each relevant far-field cube $C_{f f}$ it is checked whether the $M_{n}^{m}$ have been computed (continue with step 6 and step 5) or must be determined in the current step (step 8).

The goal of step 8 is to replace sources on boundary elements with sets of $M_{n}^{m}$. If $C_{f f}$ has child cubes at finer octree levels, the $\bar{M}_{n}^{m}$ of the child cube are transformed using again a rotation of the coordinate system to accelerate the computations

$$
M_{n}^{m}=\sum_{k=0}^{n} \bar{M}_{n-k}^{m} T_{m 2 m}(n, m, k) .
$$

At the finest octree level, eqn (6) is applied to compute the $M_{n}^{m}$ directly from the sources on the boundary elements.

In the first run, the reversed FMM algorithm has computational costs compared to the classical FMM algorithm using fast transformations of series expansions based on a rotation of the coordinate system [8]. However, during the post-processing, the FMM algorithm is executed multiple times, e.g. for each point of a field line. Since the reversed FMM algorithm starts at the evaluation point $\boldsymbol{r}_{e p}$, steps 1 to 4 are executed in most cases, only. In some cases, step 5 is additionally required. Steps 6 and 7 are needed only during the first run of the reversed FMM algorithm. Hence, the computational costs are in total significantly reduced by my reversed FMM algorithm compared to the classical FMM algorithm for postprocessing.

\subsection{Numerical properties of the local expansion}

As I have shown in Section 2.3, the local expansion is very well suited to approximate scalar functions within a limited domain using appropriately chosen complex series coefficients $L_{n}^{m}$. Here, I apply the truncated local expansion (7) to the test configuration depicted in Fig. 6. A linear triangular Lagrange boundary element is used exemplarily to describe single-layer sources, here a constant surface charge density of $10 \mathrm{nC} / \mathrm{m}^{2}$. The cube structure of the applied octree scheme is shown by the black lines. The part of the boundary element, which is within its assigned cube, is highlighted using green color and its parts, which are outside its cube, are marked with red color. So, I study a typical situation where the octree scheme does not perfectly fit the boundary element mesh of the problem. The field of the source element is evaluated at the surface and inside the two cubes, for which in Fig. 6 the scalar potential is shown using the depicted color scheme. Since a linear problem is studied, geometrical dimensions do not matter and a normalized edge length of the depicted cubes of $1 \mathrm{~m}$ is used.

As above shown, the FMM algorithm or the reversed FMM algorithm is complex, and the approximation error of truncated series expansions and transformations is composed of errors of many different numerical operations. Hence, the total error of the FMM is difficult to specify and measure.

Here, I start the accuracy analysis of the FMM with studies of the accuracy of eqn (7). To exclude all errors of the reversed FMM algorithm, I compute the $L_{n}^{m}$ directly by an evaluation of eqn (8) for the test element and with the origin of the local spherical coordinate systems of the cubes at the centers of the considered cubes. The potential $u$ and the electric field $\boldsymbol{E}=$ $-\nabla u$ are evaluated in a slice going through the cubes and on the cubes' surfaces. These results represent the accuracy limits of the FMM for a given order $L$. 


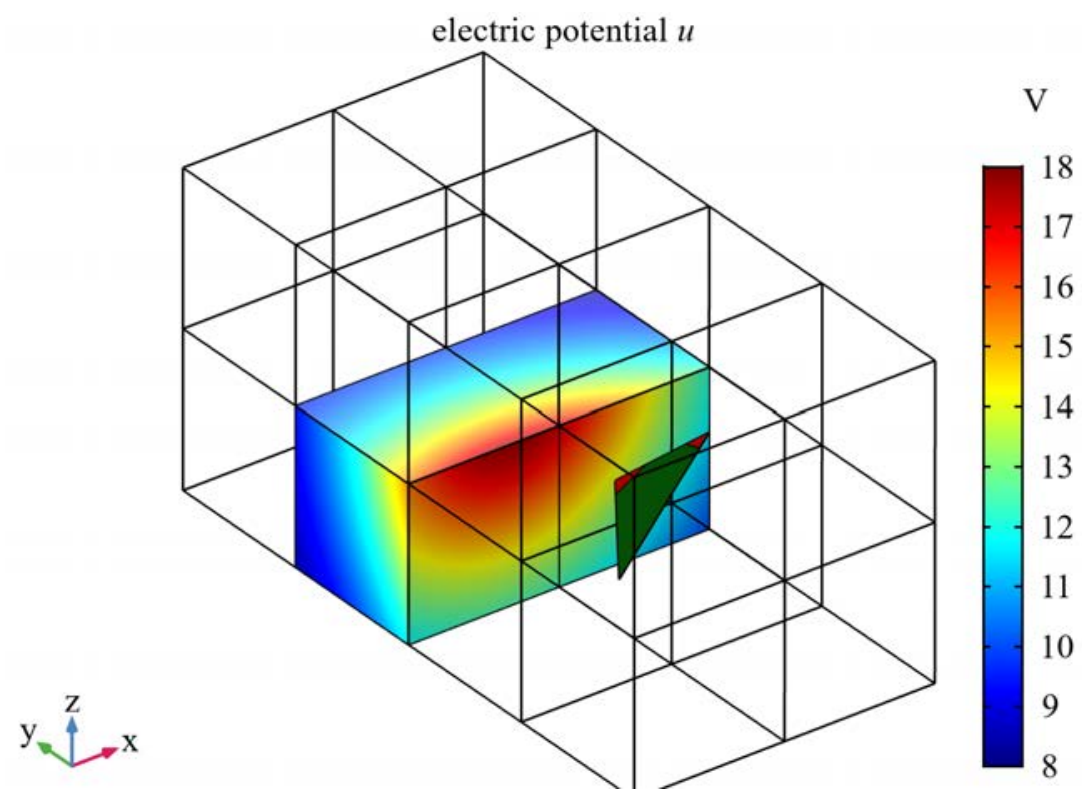

Figure 6: Test configuration for the presented accuracy analysis.

The potential $u$ at the surfaces of the two cubes using eqns (7) and (8) with a truncated order $L=5$ is depicted in Fig. 7. Furthermore, the relative error in comparison to the direct evaluation of the related BEM integral is shown. Even for a low order $L$, the color plot of $u$ is smooth and a discontinuity of $u$ at the cubes' interface is barely visible. However, the analysis of the related relative error leads to the conclusion that the discontinuity of $u$ is not negligible. This is clearly illustrated by the plot of $u$ in a slice in Fig. 8.

Of course, the relative error decreases if $L$ or the distance between the element and the considered cubes is increased. That means it is in principle possible to find a good compromise between accuracy and computational costs for a reasonable approximation of $u$. Then, the approximation error is in the range of accuracy of the BEM and discontinuities of $u$ at the cubes' interfaces are in practice negligible.
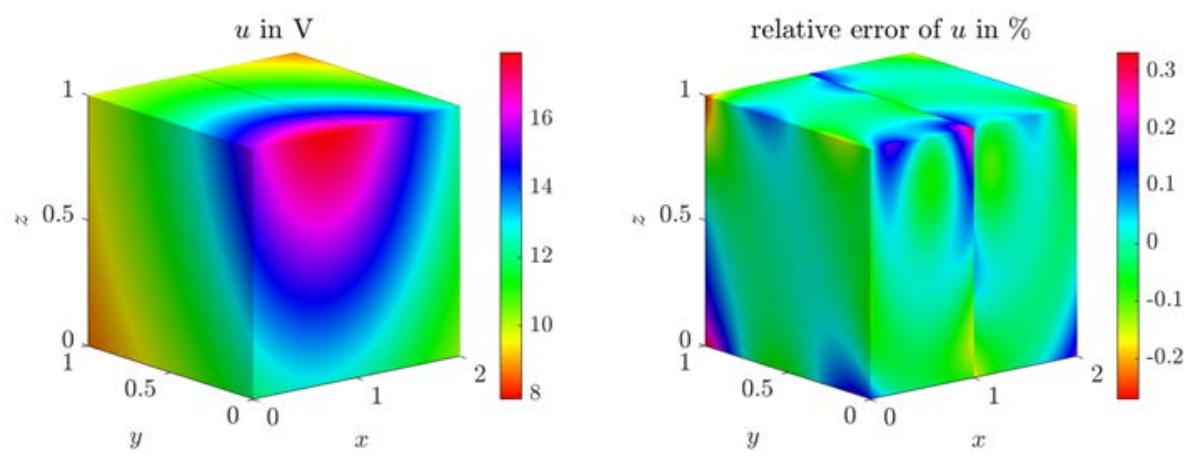

Figure 7: $\quad$ Potential $u$ computed at the cubes' surfaces using eqns (7) and (8) with $L=5$ and its relative error in comparison to direct evaluation of the BEM integral. 

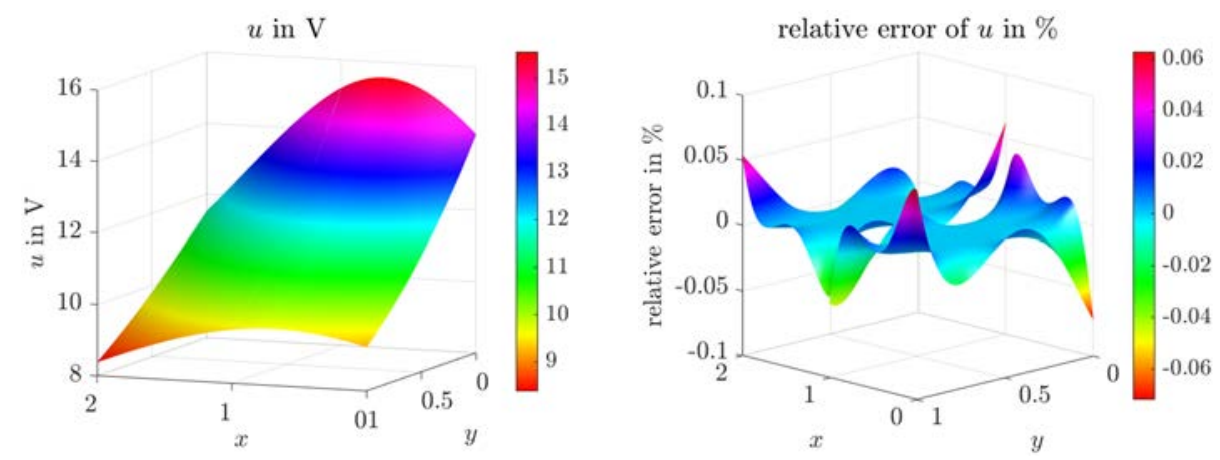

Figure 8: Potential $u$ computed at a horizontal slice using eqns (7) and (8) with $L=5$ and its relative error in comparison to direct evaluation of the BEM integral.

In most applications, not only the potential $u$ but also the electric field $\boldsymbol{E}=-\nabla u$ is of large interest for an engineer. Hence, the norm $E=\|\boldsymbol{E}\|$ and its relative error in comparison to the direct evaluation of the related BEM integrals is depicted in Fig. 9 at a horizontal slice through the considered cubes for an order $L=5$. The plot of $E$ shows a significant discontinuity and the relative error is much larger than the one of $u$. That means for precise field computations based on the derivative of the potential, a high order of the series expansions is required. Especially a visible discontinuity at the cubes' interfaces should be avoided.
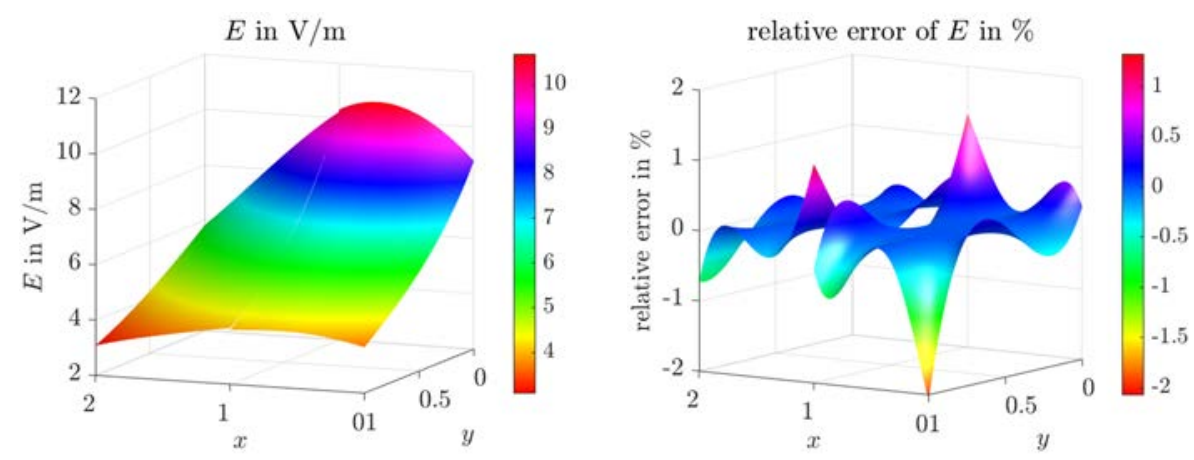

Figure 9: Electric field $E$ computed at a horizontal slice using the gradient of eqns (7) and (8) with $L=5$ and its relative error in comparison to direct evaluation of the BEM integral.

I have repeated the computations of $u$ and $E$ with an order of $L=10$ which gives in total satisfactory results for most applications. The relative error of both values is depicted in Fig. 10. For the studied configuration, the approximation error of $u$ is negligibly small and even the approximation error of $E$ has no relevant effect on the total results. Note, the approximation error can be larger if the source element has larger parts outside the related cube. Such situations often appear in the context of highly adaptive meshes. 

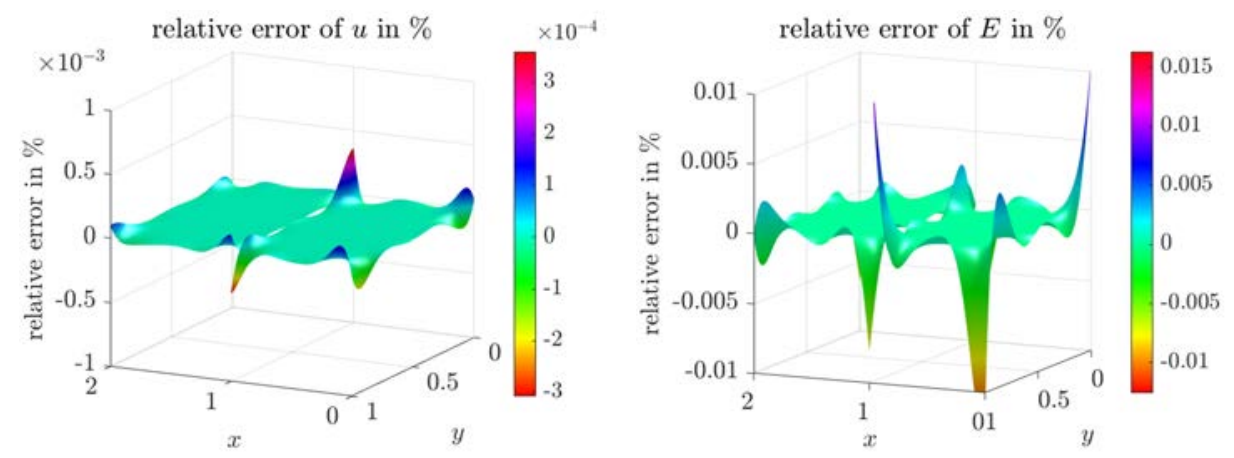

Figure 10: The relative error of $u$ and $E$ at a horizontal slice using eqn (7) and its gradient with $L=10$ in comparison to direct evaluation of the BEM integral.

In total, the local expansion results in precisely computed field values if the order of the truncated series expansion is carefully chosen. Discontinuities at cubes' interfaces are then small enough not to be relevant in practice.

\subsection{Numerical properties of the local-to-local transformation}

In the preceding section, I have shown the potential and the superb properties of the local expansion for the fast multipole method and related applications. Here, I put the focus on the local-to-local transformation (9) and its accuracy. Particularly, I want to answer the questions of how this transformation influences the total accuracy of the FMM and which parameters can be used to improve the accuracy of the FMM.

In this study, I compute the local coefficients $\tilde{L}_{n}^{m}$ of the parent cube of the two considered cubes directly using eqn (8). The relative error of the potential evaluated using eqn (7) with $\tilde{L}_{n}^{m}$ in comparison to the direct computation of the boundary integral is depicted in Fig. 11 on the left side. The order of the truncated series expansion is $L=10$. It is worth to mention, that the error at points that are close to the boundary element is relatively large. The reason is that in this test configuration the distance between the considered parent cube and the cube with the boundary element is smaller than usual in the FMM. At points in typical distance to the source element, the error is much smaller, here the points near the backside of the two considered cubes. That means in practice, the distance between the cubes which are used for the following multipole-to-local transformation and the part of the elements which sticks out of the related cube are the most important parameters to adjust the total accuracy of the FMM. The relative error of the potential using the local coefficients of the considered cubes after the local-to-local transformation is shown in Fig. 11 on the right side. This error is nearly identical to the previous one. A comparison of the potential values in both cases underlines this observation.

My conclusions of this study are that the local-to-local transformation has almost no influence on the accuracy of the FMM and the accuracy of the truncated local expansion is set by the parameters of the parent cube and not by the considered cube. The reason is that the potential inside the parent cube is described with the help of its local expansion and this series expansion is used to determine the coefficients of the local expansion of the child cubes. So to speak the transformed local expansion of the child cube is nothing else than the local expansion of the parent cube but with the shifted origin of the local coordinate system. 

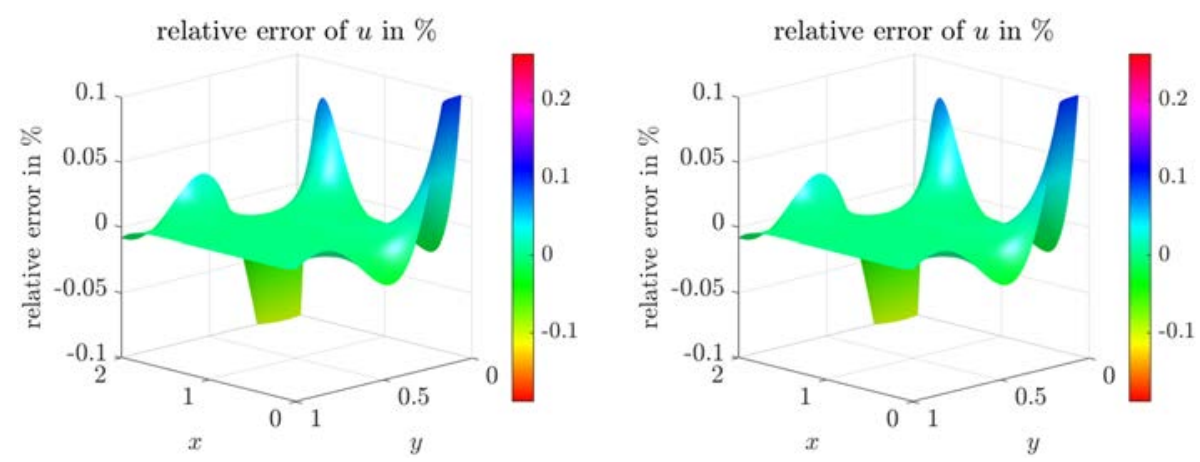

Figure 11: Relative error of the potential of the local expansion of the parent cube (left side) and the transformed local expansions (right side) both for $L=10$.

\subsection{Numerical properties of the multipole expansion}

The computation of the multipole coefficients using eqn (6) is the essential step of the FMM to convert the continuous charge distribution on the elements into multipoles up to the defined order $L$. The accuracy of the potential computed based on eqn (5) depends both on the number of multipoles and the distance between the region of the original charges and the evaluation point $\boldsymbol{r}$. Within the FMM algorithm, the multipole coefficients of a cube are transformed using the multipole-to-multipole expansion into the multipole coefficients of the related parent cube. There, the same order of multipoles is available. That means the accuracy of the multipole expansion of the parent cube is comparable to the accuracy of the multipole expansion of its child cube. Analog to the local-to-local expansion the size of the domain of the sources is changed during this transformation and the overall accuracy is decreased.

I test this situation using the setup of Fig. 6. First, I compute the multipole expansion of the cube of the boundary element and evaluate the potential inside the two evaluation cubes (Fig. 12, left side). Then, I compute the multipole expansion of the parent cube both directly using eqn (6) and via the multipole-to-multipole transformation. Again, the shift of the coordinate system does not influence the accuracy. However, the enlarged size of the source domain lowers the accuracy of the potential close to the source domain (Fig. 12 right side).
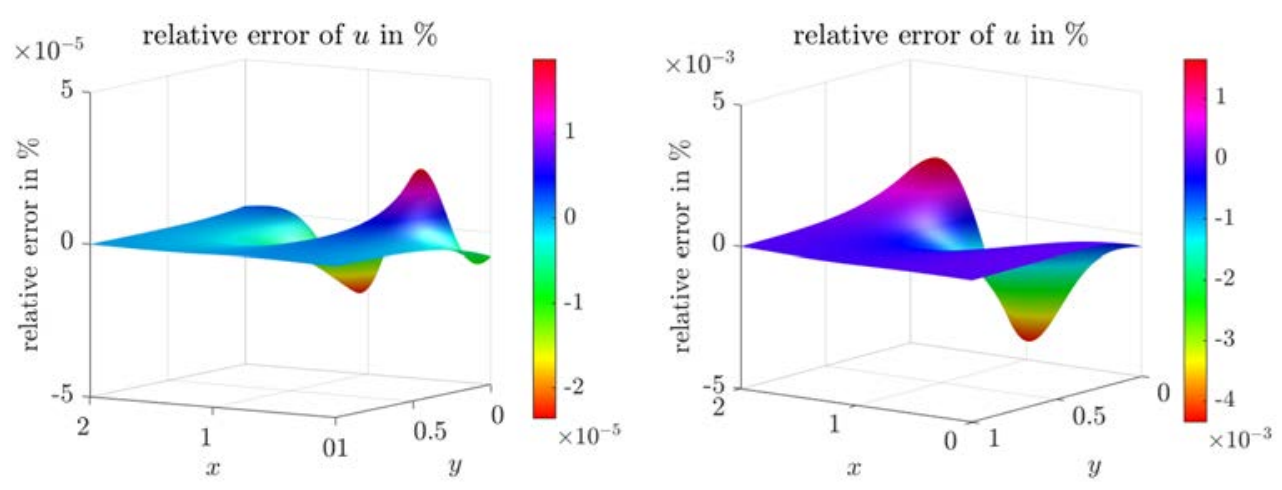

Figure 12: Relative error of the multipole expansion of the element cube (left side) and the transformed multipole expansion of the parent cube (right side) both for $L=10$. 
3.5 Numerical properties of the multipole-to-local transformation

In my last study, I compute the multipole coefficients $M_{n}^{m}$ of the cube of the source element using eqn (6). Then, I apply the multipole-to-local transformation to get the local coefficients $L_{n}^{m}$ of the two evaluation cubes. The relative error of the potential $u$ using the related local expansions in the cubes is depicted for $L=5$ and $L=10$ in Fig. 13. A comparison with Figs 8 and 10 shows that the multipole-to-local transformation has no visible influence on the accuracy of the FMM.
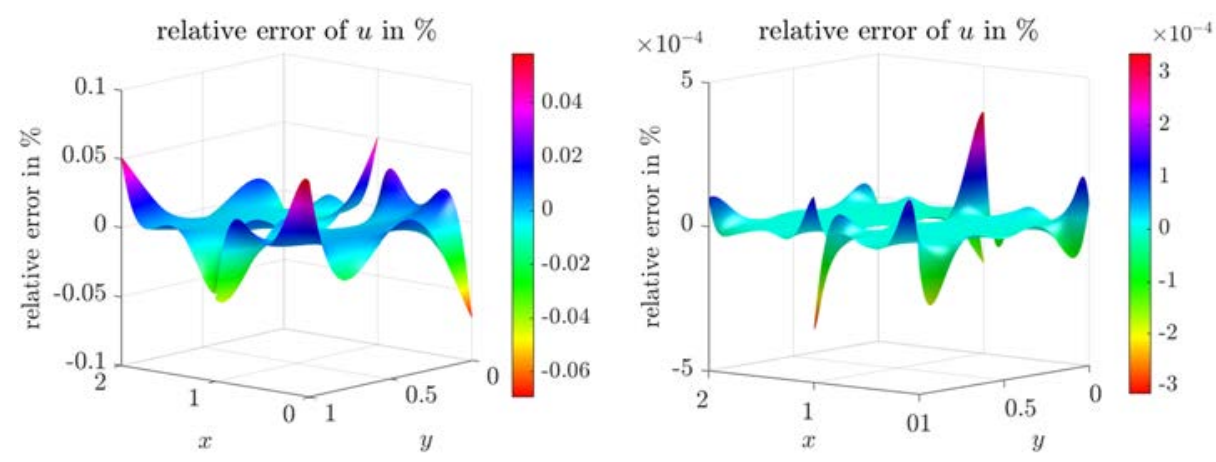

Figure 13: Relative error of the potential of the local expansion computed using the multipole-to-local transformation for $L=5$ and $L=10$.

\section{CONCLUSIONS}

In summary, my here presented studies on spherical harmonics and the fast multipole method deepen the understanding of both and therefore support more efficient use of these powerful tools. First, I have revisited spherical harmonics and particularly showed that spherical harmonics even for high degrees have coordinate dependencies in Cartesian coordinates although their input arguments are defined in spherical coordinates. Therefore, the coefficients of the related truncated series expansion enable accurate control of the intended approximation concerning dependencies in Cartesian coordinates. Then, I have discussed the reversed FMM algorithm in detail and theoretically showed its efficiency in the context of meshfree post-processing. Finally, I have demonstrated based on an example relevant to practice that with typical FMM parameters highly accurate computations are possible with the FMM. I could experimentally prove that the influence of transformations of multipole and local coefficients is negligible. The total accuracy of the FMM is mainly influenced by distances between the related octree cubes and the actual distance between boundary elements.

\section{REFERENCES}

[1] Greengard, L. \& Rokhlin, V., The rapid evaluation of potential fields in three dimensions. Vortex Methods, Lecture Notes in Mathematics, vol. 1360, eds C. Anderson \& C. Greengard, Springer, 1988. DOI: 10.1007/BFb0089775.

[2] Nabors, K. \& White, J., FastCap: A multipole accelerated 3-D capacitance extraction program. IEEE Transactions on Computer-Aided Design of Integrated Circuits and Systems, 10(11), pp. 1447-1459, 1991. DOI: 10.1109/43.97624. 
[3] Buchau, A., Rieger, W. \& Rucker, W.M., BEM computations using the fast multipole method in combination with higher order elements and the Galerkin method. IEEE Transactions on Magnetics, 37(5), pp. 3181-3185, 2001. DOI: 10.1109/20.952572.

[4] Buchau, A., Rucker, W.M., Rain, O., Rischmüller, V., Kurz, S. \& Rjasanow, S., Comparison between different approaches for fast and efficient 3-D BEM computations. IEEE Transactions on Magnetics, 39(3), pp. 1107-1110, 2003. DOI: 10.1109/TMAG.2003.810167.

[5] Brebbia, C.A., Telles, J.C.F. \& Wrobel, L.C., Boundary Element Techniques, Springer-Verlag: Berlin and New York, 1984.

[6] Buchau, A., Precise and robust magnetic field computations for high-end smart sensor applications. WIT Transactions on Engineering Sciences, vol. 126, WIT Press: Southampton and Boston, pp. 75-87, 2019. DOI: 10.2495/BE420071.

[7] Buchau, A. \& Rucker, W.M., Meshfree computation of field lines across multiple domains using fast boundary element methods. IEEE Transactions on Magnetics, 51(3), pp. 1-4, 2015. DOI: 10.1109/TMAG.2014.2359520.

[8] Greengard, L. \& Rokhlin, V., A new version of the fast multipole method for the Laplace equation in three dimensions. Acta Numerica, 6, pp. 229-269, 1997.

DOI: $10.1017 / \mathrm{S} 0962492900002725$.

[9] Arfken, G.B. \& Weber, H.J., Mathematical Methods for Physicists, Academic Press, 1995.

[10] Hobson, E.W., The Theory of Spherical and Ellipsoidal Harmonics, Cambridge University Press, 1931.

[11] Maxwell, J.C., Treatise on Electricity and Magnetism, Clarendon Press, 1873.

[12] Buchau, A. \& Rucker, W.M., Feasibility of a meshfree post-processing for boundary element methods. WIT Transactions on Modelling and Simulation, vol. 61, WIT Press: Southampton and Boston, pp. 327-338, 2015. DOI: 10.2495/BEM380261. 